\title{
Aggressive fibromatosis response to tamoxifen: lack of correlation between MRI and symptomatic response
}

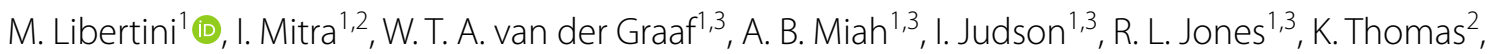 \\ E. Moskovic ${ }^{1,3}$, Z. Szucs $^{1}$, C. Benson ${ }^{1}$ and C. Messiou ${ }^{1,2,3^{*}}$
}

\begin{abstract}
Background: One of the commonly used systemic agents for the treatment of aggressive fibromatosis is the antioestrogen drug tamoxifen. However, data on efficacy and optimum methods of response assessment are limited, consisting mainly of small case series and reports.

Methods: A retrospective database was used to identify consecutive patients diagnosed with aggressive fibromatosis (AF) and treated with tamoxifen plus/minus non-steroidal anti-inflammatory drugs at our tertiary referral centre between 2007 and 2014. MRI and symptom changes were recorded.

Results: Thirty-two patients (13 male 19 female, median age 41 years) were included. Median duration of treatment with tamoxifen was 316 days. Of 9 patients with progressive disease by RECIST 1.1 (28\%): 4 patients experienced worsening symptoms; 3 patients had improved symptoms and 2 had no change in symptoms. Of 22 patients with stable disease (69\%): 11 had no change in symptoms; 6 had improved symptoms and 5 patients had worsening symptoms. One patient achieved a partial response with improved symptoms.

Conclusions: No relationship was identified between symptomatic benefit and response by RECIST 1.1 on MRI. Prospective studies in AF should incorporate endpoints focusing on patient symptoms.
\end{abstract}

Keywords: Aggressive fibromatosis, Anti-oestrogen therapy, Symptoms, MRI features, T2 weighted signal

\section{Background}

Aggressive fibromatosis (AF), also named desmoid-type fibromatosis, is characterised by monoclonal myofibroblastic proliferation in soft tissues. It is a rare disease accounting for $3 \%$ of all soft tissue neoplasms, with an incidence of $2-5$ people per million per year [1]. It has a female predominance and a peak incidence in the third to fourth decades [2]. AF is often sporadic, however, there is a reported increased incidence of $3.5-32 \%$ in patients with familial adenomatous polyposis (FAP) or Gardner's variant [3, 4]. AF is usually solitary but multifocal tumours have been reported [5]. It may arise

\footnotetext{
*Correspondence: Christina.Messiou@rmh.nhs.uk

2 Department of Radiology, The Royal Marsden NHS Foundation Trust, 203

Fulham Road, London SW3 6JJ, UK

Full list of author information is available at the end of the article
}

from any anatomical site, commonly the extremities, abdominal and chest wall and paravertebral tissues $[6,7]$. Although AF is slow growing without metastatic potential, its unpredictable behaviour, propensity for progressive infiltration and local invasion makes treatment challenging. Currently there is no established evidencebased approach to treatment [8], although a consensus approach based on wide consultation with physicians and patient groups has been published [9]. Active surveillance is now often used in asymptomatic cases [10]. High local recurrence rates of $15-50 \%$ [11-14] up to $87 \%$ [15] in younger patients, despite apparently complete resection, have reduced the popularity of surgical resection as initial management. Radiotherapy can help improve local control [16] however, side effects, including radiationinduced malignancies have to be considered especially in young patients [17].

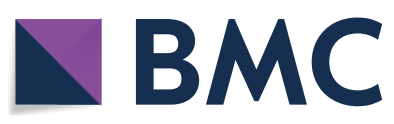

(c) The Author(s) 2018. This article is distributed under the terms of the Creative Commons Attribution 4.0 International License (http://creativecommons.org/licenses/by/4.0/), which permits unrestricted use, distribution, and reproduction in any medium, provided you give appropriate credit to the original author(s) and the source, provide a link to the Creative Commons license, and indicate if changes were made. The Creative Commons Public Domain Dedication waiver (http://creativecommons.org/ publicdomain/zero/1.0/) applies to the data made available in this article, unless otherwise stated. 
Drugs used in the treatment of AF, include hormonal therapy (e.g. tamoxifen and toremifene) [18], Non steroidal antinflammatory drugs (NSAIDs) and cytotoxic chemotherapeutic agents, such as anthracycline-based regimens [19] (including pegylated liposomal doxorubicin [20]) and vinblastine plus methotrexate [21]. Tyrosine kinase inhibitors, including imatinib [22], sorafenib [23] and pazopanib [24] can also play a role in the treatment of AF. A recent phase I study demonstrated demonstrated promising efficacy of a $\gamma$-secretase inhibitor in desmoid tumours [25].

For many centres, first line systemic treatment of nonresectable or symptomatic desmoid-type fibromatosis consists of hormonal manipulation, with or without a NSAID. Particularly in centres within the United Kingdom, this is heavily influenced by lack of reimbursed alternatives, availability and the low side effect profile. Immunohistochemical studies have demonstrated the presence of oestrogen receptor-beta in $90 \%$ of desmoidtype tumours [26]. This is supported by the tendency of fibromatosis to occur more often in women, particularly during pregnancy/within 1 year post partum [27], or on oral contraception, and there are reports of spontaneous regression during menopause and post-partum [2, 28]. Several publications have documented the effectiveness of hormonal manipulation in AF treatment [29-32]. Despite the lack of randomised prospective data, it has been reported that antioestrogen therapy can be effective in about half of patients [18]. Tamoxifen is a nonsteroidal triphenylethylene derivative that binds to oestrogen receptors. One suggested mechanism for the anti-proliferative action of tamoxifen is regulation of the synthesis of the cytokine transforming growth factor- $\beta$ (TGF- $\beta$ ) [32] and its receptors, which are also involved in AF pathogenesis.

There are several means of monitoring treatment response, including clinical evaluation of tumour size and symptoms as well as radiological. The Response Evaluation Criteria in Solid Tumours (RECIST 1.1) [33] are currently employed within clinical trials. Magnetic resonance imaging (MRI) has become the imaging modality of choice for soft tissue lesions, due to better evaluation of the tumour and its relationship with surrounding structures such as nerves and vessels. Given the chronic nature of the disease, the lack of radiation exposure makes MRI ideal for follow up studies. Furthermore, in lesions undergoing radiation or drug therapy, MR surveillance has been used to assess response to treatment with a decrease in T2-weighted signal and lesion size being suggested as indicators of treatment response [34].

The variable content of spindle cells, collagen and myxoid tissue of AF correlates with the observation that these lesions often show heterogeneous signals on MRI [35]. In particular, the highly cellular, actively growing lesions tend to be of high signal on T2-weighted MR images $[6,36,37]$. Interspersed low signal bands correspond with the collagen bundles. As the lesion matures, the increase in collagen deposition and decreased cellularity result in a decrease in T2 signal $[38,39]$. However to date it has not been possible to predict behaviour based on MRI signal [40, 41].

The main aims of this study were to assess MRI response and symptom control in patients with $\mathrm{AF}$ treated with tamoxifen with or without NSAIDs.

\section{Methods}

\section{Patient selection}

The prospectively collected Royal Marsden Hospital sarcoma database was used to identify consecutive patients diagnosed with AF and treated with tamoxifen at our tertiary referral centre between 2007 and 2014. Institutional approval was obtained. Inclusion criteria were patients aged 18 years and over, treated with tamoxifen, with a baseline and at least one follow-up MRI scan. Demographic data, disease characteristics, previous treatments, date of starting and stopping tamoxifen, toxicity and clinical symptoms were collected from clinical notes. Descriptive statistical analysis was applied: progression-free survival (PFS) was calculated using the Kaplan-Meier method.

\section{Imaging data}

Baseline MRI images, defined as the last MRI performed prior to tamoxifen treatment, and subsequent follow up MRI images were identified for each patient. Where available a pre-baseline MRI was also collected. On average 3 (range 2-6) follow up MRIs were assessed for each patient. The minimum MRI protocol for inclusion in the study was axial T1W, T2W, STIR and coronal T2W and STIR images. All images were re-reviewed by a specialist soft tissue radiologist (CM). Tumour size, RECIST 1.1 assessment and T2-weighted signal changes were documented at each time point.

\section{Results}

Between 2007 and 2014 a total of 35 patients were treated with tamoxifen at the Royal Marsden Hospital. Baseline imaging was not available for 3 patients, and they were therefore not eligible for this study. Of the remaining 32 cases, the median age at the time of commencing tamoxifen was 41 years (range 19-68 years). There was a 3:2 female to male ratio [19 (60\%): $13(40 \%)]$. One patient (3\%) had a diagnosis of FAP. The most common site of origin was limb and limb girdle (18; 56\%), followed by chest wall $(5 ; 15 \%)$, pelvis $(3 ; 10 \%)$, abdominal wall $(3$; $10 \%)$, paravertebral tissues $(1 ; 3 \%)$ and head/neck $(2 ; 6 \%)$.

Fourteen patients (44\%) received tamoxifen as first-line treatment. Eighteen of 32 patients (56\%) had been treated 
previously, with surgery, radiotherapy, steroid injections, NSAIDs or doxorubicin chemotherapy. Patient and disease characteristics are summarised in Table 1.

Tamoxifen treatment was started due to tumour growth and worsening symptoms, mostly characterised by pain, including neuropathic and somatic pain, and decreased range of movement. Four patients (12\%) were asymptomatic at the time of starting tamoxifen.

The average length of time on tamoxifen was 316 days, ranging from 1 month to 3 years (33-997 days). Tamoxifen dosages used were $40 \mathrm{mg}$ (15 patients), $20 \mathrm{mg}$ (15 patients), $10 \mathrm{mg}$ (1 patient) and unknown (1 patient). Twenty-four patients (75\%) received tamoxifen in association with a NSAID (naproxen or diclofenac). Thirteen of the 32 patients (41\%) suffered from tamoxifen-related side effects, most commonly hot flushes and mood swings. Two patients had their $40 \mathrm{mg}$ dose reduced to $20 \mathrm{mg}$ due to side effects. One of those was a 40-year old male who experienced increased tiredness and the other a 27-year old male who suffered from mood swings, fatigue and hot flushes.

The most common reasons for stopping tamoxifen were tumour progression (10,31\%), and grade $2-3$ side effects $(8,25 \%)$, such as hot flushes, mood swings and fatigue. Four patients (13\%) stopped due to lack of any perceived benefit and $3(9 \%)$ due to worsening symptoms. One patient with stable disease stopped tamoxifen as she was planning on starting a family and she was aware of possible associated birth defects [42]. One patient stopped

Table 1 Patient and disease characteristics

\begin{tabular}{lcll}
\hline & $\#$ & $(\%)$ & [range] \\
\hline Number of patients & 32 & & \\
Male & 14 & $(40 \%)$ & \\
Female & 19 & $(60 \%)$ & [19-68] \\
Median age on starting tamoxifen & 41 & & \\
Site of origin & & & \\
$\quad$ Extremities (including girdle) & 18 & $(56 \%)$ \\
Chest wall & 5 & $(15 \%)$ \\
Pelvis & 3 & $(10 \%)$ \\
Abdominal wall & 3 & $(10 \%)$ \\
Paravertebral & 1 & $(3 \%)$ \\
$\quad$ Head and neck & 2 & $(6 \%)$ \\
Sporadic disease & 31 & $(97 \%)$ \\
FAP-associated & 1 & $(3 \%)$ \\
Previous treatments & 18 & $(56 \%)$ \\
$\quad$ Local treatment & & \\
$\quad$ Surgery & 10 & $(31 \%)$ \\
$\quad$ Surgery and RT & 7 & $(21 \%)$ \\
$\quad$ RT alone & 0 & \\
$\quad$ Systemic treatment & 4 & $(12 \%)$ \\
No previous treatment & 14 & $(44 \%)$ \\
\hline
\end{tabular}

tamoxifen because of pregnancy. Two patients died for reasons unrelated to AF. One patient stopped tamoxifen due to side effects, and subsequently received an antitumour necrosis factor (anti-TNF)- $\alpha$ agent, adalimumab [43], for his rheumatoid arthritis and the AF decreased in size. Two patients continue on tamoxifen.

Tamoxifen with or without a NSAID, resulted in symptom improvement in 10 patients (31\%) -5 patients on $40 \mathrm{mg}$ tamoxifen, 4 patients on $20 \mathrm{mg}$ tamoxifen and 1 patient on $10 \mathrm{mg}$ tamoxifen (8 of these 10 patients were also taking NSAIDs). Worsening symptoms were experienced by 9 patients $(28 \%)-2$ patients on $40 \mathrm{mg}$ tamoxifen, 6 patients on $20 \mathrm{mg}$ tamoxifen and 1 unknown $(6$ of these 9 patients were also taking NSAIDs). Thirteen patients did not experience any change in symptoms (41\%) - 9 patients on $40 \mathrm{mg}$ tamoxifen, 4 patients on $20 \mathrm{mg}$ tamoxifen (10 of these 13 patients were also taking NSAIDs). The majority of patients with symptomatic benefit did not have significant changes in size or signal on MRI (Fig. 1a, b).

The median tumour size on starting tamoxifen was $60 \mathrm{~mm}$ (range 23-165 mm). There was a varied response in tumour size. Eighteen patients (56\%) had increase in tumour size, 6 patients (19\%) had a reduction in tumour size and 8 patients $(25 \%)$ had no change in tumour size. By RECIST 1.1, 9 patients (28\%) had progressive disease -6 patients on $40 \mathrm{mg}$ tamoxifen, 2 patients on $20 \mathrm{mg}$ tamoxifen and 1 unknown; 22 patients (69\%) had stable disease -8 patients on $40 \mathrm{mg}$ tamoxifen, 13 patients on $20 \mathrm{mg}$ tamoxifen, 1 patient on $10 \mathrm{mg}$ tamoxifen and 1 patient (3\%) had a partial response-40 mg tamoxifen. Median progression-free survival (PFS) per RECIST 1.1 was 10 months with (95\% CI 6.4-24.6); PFS at 1 and 2 years was $50 \%$ (95\% CI 32-66) and 34\% (95\% CI 19-51), respectively (Fig. 2), with a median follow-up of 45.5 months (range 14-105).

We divided our series into three RECIST 1.1 criteriabased groups: progressive disease, stable disease and partial response. Of 9 patients with progressive disease (28\%): 4 patients experienced worsening symptoms with increased pain; 3 patients had an improvement in pain and increase in range of movement and no change in symptoms was observed in the remaining 2 patients. T2 signal increase was observed in 1 case, which correlated with clinical deterioration; in 8 of 9 cases there was no change in T2 signal. Of 22 patients with stable disease (69\%): 11 had no change in symptoms with T2 signal reduction in 3 cases and increase in 1; 6 experienced symptom improvement: 1 of 6 had a correlating T2 signal reduction. Five patients complained of worsening symptoms without any change in T2 signal.

One patient achieved a partial response (3\%). This patient was a 35 -year old male affected by AF involving 

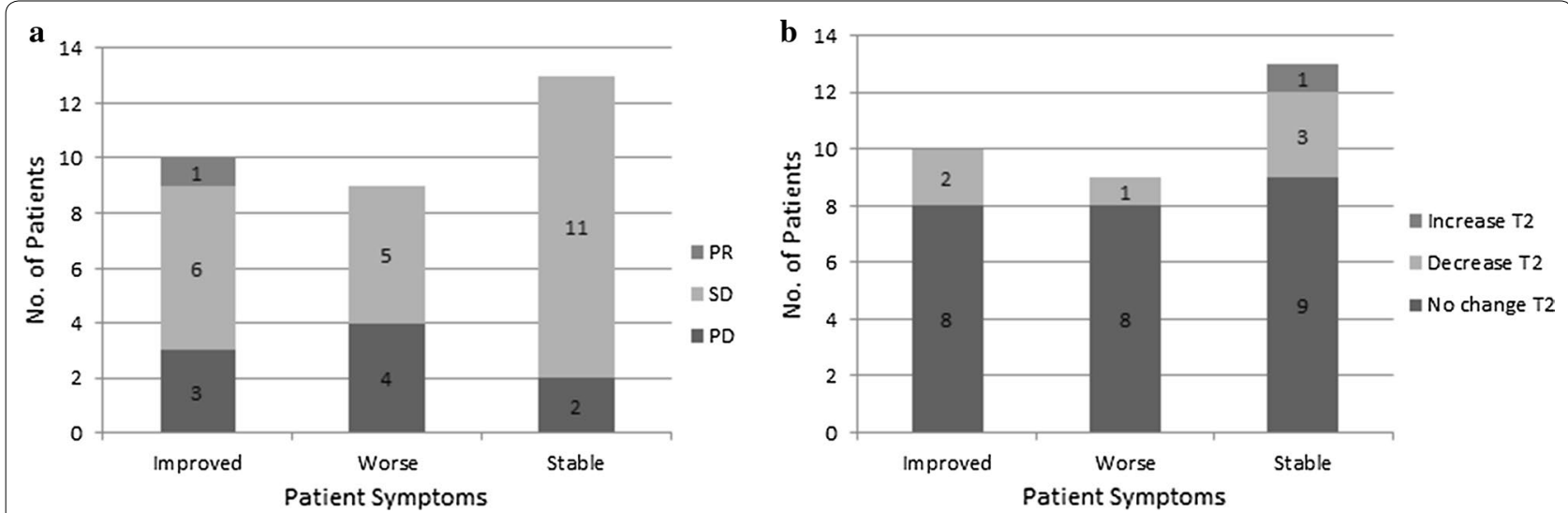

Fig. 1 Barcharts of patient symptoms and corresponding RECIST 1.1 status (a) and T2W MRI signal changes (b)

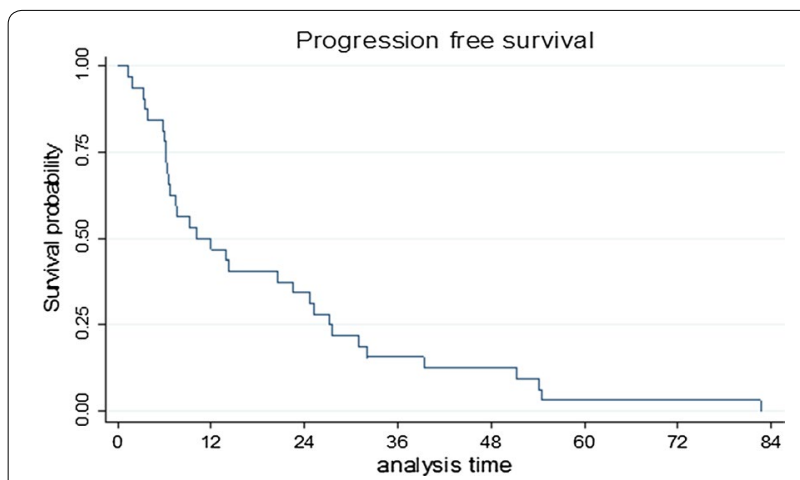

Fig. 2 Kaplan-Meier progression free survival analysis after treatment with tamoxifen. Median PFS was 10 months (95\% Cl 6.4-24.6)

the right anterior abdominal wall with abdominal pain at baseline. This patient is still on treatment, and has completed 476 days of therapy at the time of analysis. After 18 months of treatment, he experienced an improvement in symptoms with a reduction in pain. The symptomatic improvement corresponded with a decrease in size and T2 signal on MRI (Fig. 3).

Additional pre-baseline MRI scans were available for 14 patients. Thirteen out of 14 patients' tumours were increasing in size prior to starting tamoxifen. Six continued to increase following tamoxifen; 5 showed some decrease in size; 2 became stable having demonstrated growth prior to starting tamoxifen.

\section{Discussion}

Aggressive fibromatosis is a challenging disease with an unpredictable behaviour. The unsatisfactory outcomes of surgery and the fact that growth arrest and regression can occur spontaneously have led to the increased adoption of active surveillance as the initial approach to management [44]. However hormonal manipulation has been commonly used particularly in the United Kingdom as first-line systemic therapy in AF [10].

Our study of 32 patients is limited by the retrospective design and the collection of symptomatic changes by
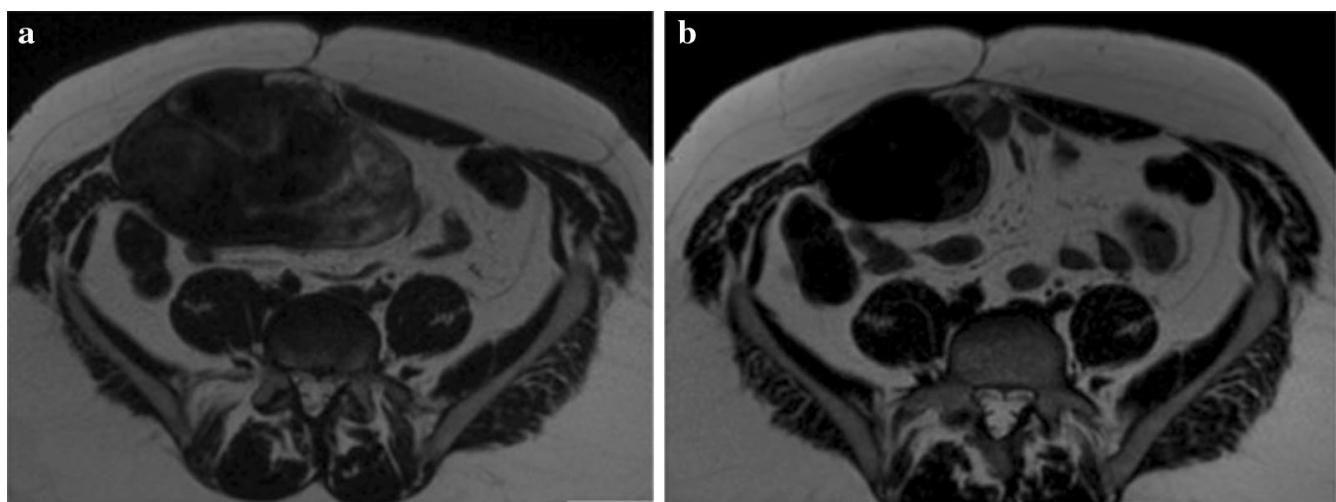

Fig. 3 Axial T2 weighted MRI images showing right anterior abdominal wall fibromatosis in a 35-year old male (a) and the corresponding MRI after 7 months of treatment with tamoxifen (b) show decrease in size and T2 signal 
retrospective case note review rather than prospective dedicated questionnaires. However, to our knowledge, this is the largest single series evaluating the relationship between symptoms and MRI response in patients with AF treated with tamoxifen with/without NSAIDs.

Within this case series, 14 of the 32 patients (44\%) received tamoxifen as first-line treatment. This could reflect the complexity of the cases referred to our tertiary centre but also the focus on preserving function and quality of life. Furthermore, we found that 8 of the 32 patients $(25 \%)$ had to stop tamoxifen due to side effects, which is higher than the previously reported rate of $10 \%$ [45] and remarkable given the, for this condition, relatively low dose of tamoxifen prescribed in our patients $[29,46]$.

In our series 18 of the 32 patients (56\%) had an increase in tumour size and among these, $28 \%$ were defined as disease progression by RECIST 1.1. This is in keeping with the rates of disease progression stated in a systematic review [18]. However, our stable disease and partial response rates of 69 and 3\% respectively, do not correlate with the 18 and $58 \%$ rates previously quoted, raising the possibility of an overestimation in the reported efficacy of tamoxifen in AF. Importantly, 31\% of patients did report symptomatic benefit, although the positive contribution of NSAIDs cannot be excluded. Seventy-five percent of cases in this series were treated with concomitant NSAIDs, which may have influenced the results, since prostaglandin blockage has been shown to provide some benefit in the treatment of AF [47].

Interestingly, one patient treated with a fully human monoclonal antibody tumor necrosis factor inhibitor (TNFi), adalimumab, used for rheumatoid arthritis showed a response after tamoxifen discontinuation, suggesting either a possible late tamoxifen effect or a role of immunomodulation in AF pathogenesis and treatment.

Among the population of patients with stable disease, representing the most heterogeneous group, we found a discordance between clinical symptoms and MRI T2 changes. This could be explained by the fact that cases with a slight increase/decrease in size are included within the category of RECIST 1.1 stable disease.

Although MRI is accepted to be the best imaging modality for visualising AF [38] this study suggests that it is less useful in demonstrating therapeutic benefit on tamoxifen \pm NSAIDs. This study has not demonstrated a clear relationship between MRI features (size/signal) and reported symptoms. This limitation may not be restricted to assessment of tamoxifen effects as Sheth et al. also reported that RECIST were not sensitive to clinically determined response in 23 patients treated with a variety of local and systemic therapies [48]. Although there is thought provoking evidence that FDG PET/CT gives some early indication of response in patients treated with imatinib, the risk:benefit ratio of the radiation doses involved must be given careful consideration particularly where multiple assessments for non malignant pathology are performed, especially in young patients [49].

Our study suggests that symptoms are arguably the most important indicators of response to tamoxifen in patients with AF. According to this observation, the incorporation of prospective validated pain scores and functional assessment tools into the evaluation of treatment in this disease would give a better indication of therapeutic benefit. This is particularly critical for the design of prospective AF studies where we suggest that endpoints should focus on patient symptoms. However, some experience with MRI in patients treated on pazopanib indicate that MRI may be more useful in assessing response to other agents [25].

For those patients with previous pre-baseline images available for comparison, a few cases showed a decrease in the rate of tumour growth. However, it is difficult to extrapolate definitive conclusions from these data because the number of patients with pre-baseline images was low and AF is known to have such a varied natural history with prolonged periods of stabilisation and eventual regression in nearly $28 \%$ of cases in one reported series [50].

\section{Conclusions}

We showed for the first time that symptomatic benefit, MRI T2 signal changes and tumour size correlate poorly. Therefore, MRI is of limited value in assessing therapeutic benefit in patients treated with tamoxifen \pm NSAIDs. This highlights the importance of robust systems to collect data on patients' symptoms and quality of life.

\section{Authors' contributions}

Concept and design (CM, IM, ML, CB, KT, IJ, AM, RJ, EM, ZS, WG); Data collection (CM, IM, ML); Data Analysis (ML, KT). Writing and interpretation (All authors). All authors read and approved the final manuscript.

\section{Author details \\ ${ }^{1}$ Sarcoma Unit, Royal Marsden NHS Foundation Trust, London, UK. ${ }^{2}$ Depart- ment of Radiology, The Royal Marsden NHS Foundation Trust, 203 Fulham Road, London SW3 6JJ, UK. ${ }^{3}$ Institute of Cancer Research, Sutton, UK. \\ Acknowledgements \\ We acknowledge National Health Service funding to the National Institute for Health Research Biomedical Research Centre, Clinical Research Facility in Imag- ing and the Cancer Research Network. The views expressed in this publication are those of the author(s) and not necessarily those of the National Health Ser- vice, the National Institute for Health Research or the Department of Health. Presented at 21 th Connective Tissue Oncology Society Annual Meeting, 2016, Lisbon, Paper 033.}

\section{Competing interests}

The authors declare that they have no competing interests.

Availability of data and materials

The datasets used and/or analysed during the current study are available from the corresponding author on reasonable request. 


\section{Consent for publication}

Not applicable.

\section{Ethics approval and consent to participate}

Institutional review board approval has been obtained from The Royal Marsden Hospital Committee for Clinical Research.

\section{Funding}

Michela Libertini received support from European School of Oncology (ESO) within the ESO training programme 2016. All authors were supported by NHS funding to the NIHR Biomedical Research Centre at the Royal Marsden Hospital.

\section{Publisher's Note}

Springer Nature remains neutral with regard to jurisdictional claims in published maps and institutional affiliations.

Received: 20 January 2018 Accepted: 4 April 2018

Published online: 14 May 2018

\section{References}

1. Van Broekhoven DL, Verhoef C, Grünhagen DJ, et al. Prognostic value of CTNNB1 gene mutation in primary sporadic aggressive fibromatosis. Ann Surg Oncol. 2015:5:1464-70.

2. Shields CJ, Winter DC, Kirwan WO, Redmond HP. Desmoid tumours. Eur J Surg Oncol. 2001;27:701-6.

3. Clark SK, Philips RK. Desmoids in familial adenomatous polyposis. Br J Surg. 1996:83:1494-504.

4. Bertario L, Russo A, Sala P, Varesco L, Giarola M, Mondini P, et al. Hereditary colorectal tumour registry. Multiple approach to the exploration of genotype-phenotype correlations in familial adenomatous polyposis. J Clin Oncol. 2003;21:1698-707.

5. Sundaram M, Duffrin H, McGuire MH, Vas W. Synchronous multicenteric desmoids tumors (aggressive fibromatosis) of the extremities. Skeletal Radiol. 1988;17:16-9.

6. Shingare $A B$, Ramaiya NH, Jagannathan JP, Krajewski KM, Giardino AA, Butrynski JE, Raut CP. A to Z of desmoid tumours. AJR. 2011;197:W1008-14.

7. Goldblum JR, Fletcher JA. Desmoid-type fibromatosis. In:Fletcher CDM, Bridge JA, Hogendoorn PCW, Mertens F, editors. World Health Organization classification of tumours of softtissue and bone. Lyon: IARC Press; 2013. p. 72-3.

8. Kasper B. Systemic treatment approaches for sporadic desmoids-type fibromatosis: scarce evidence and recommendations. Oncol Res Treat. 2015;38:244-8.

9. Kasper B, Baumgarten C, Garcia J, et al. An update on the managment of sporadic desmoid-type fibromatosis:a European Consensus Initiative between Sarcoma PAtients EuroNet (SPAEN) and European Organisation for Research and Treatment of Cancer (EORTC)/Soft Tissue and Bone Sarcoma Group (STBSG). Ann Oncol. 2017:28:2399-408.

10. Gronchi A, Colombo C, Le Péchoux C, et al. Sporadic desmoid-type fibromatosis: a stepwise approach to a non-metastasising neoplasm-A position paper from the Italian and the French Sarcoma Group. Ann Oncol. 2014;25:578-83.

11. Reitamo JJ, Sheinin TM, Pekka H. The desmoid syndrome. New aspects in the cause, pathogenesis and treatment of the desmoid tumor. Am J Surg. 1986;15:230-7.

12. Alman BA, Pajerski ME, Diaz-Cano S, Corboy K, Wolfe HJ. Aggressive fibromatosis (desmoid tumor) is a monoclonal disorder. Diagn Mol Pathol. 1997;6:98-101.

13. Merchant NB, Lewis JJ, Woodruff JM, Leung DH, Brennan MF. Extremity and trunk desmoid tumors: a multifactorial analysis of outcome. Cancer 1999;86:2045-52

14. Lev $D$, Kotilingam $D$, Wei $C$, et al. Optimizing treatment of desmoid tumors. J Clin Oncol. 2007;25:1785-91.

15. Romero JA, Kim EE, Kim CG, Chung WK, Isiklar I. Different biologic features of desmoids tumours in adult and juvenile patients: MR demonstration. J Comput Assist Tomogr. 1995;19:782-7.
16. Keus R, Nout RA, Blay JY, et al. Results of a phase II pilot study of moderate dose radiotherapy for inoperable desmoid-type fibromatosis-an EORTC STBSG and ROG study (EORTC 62991-22998). Ann Oncol. 2013;24:2672-6.

17. Guadagnolo BA, Zagars GK, Ballo MT. Long-term outcomes for desmoid tumors treated with radiation therapy. Int J Radiat Oncol Biol Phys. 2008;71:441-7.

18. Bocale D, Rotelli MT, Cavallini A, Altomare F. Anti-oestrogen therapy in the treatment of desmoids tumours: a systematic review. Colorectal Dis. 2011:13:e388-95.

19. Garbay D, Le Cesne A, Penel N, et al. Chemotherapy in patients with desmoid tumors: a study from the French Sarcoma Group (FSG). Ann Oncol. 2012;23:182-6.

20. Constantinidou A, Jones RL, Scurr M, Al-Muderis O, Judson I. Pegylated liposomal doxorubicin, an effective, well-tolerated treatment for refractory aggressive fibromatosis. Eur J Cancer. 2009;45:2930-4.

21. Park K-H, Choi YJ, Kim KW, Ro KH, Kang CH, Song SH, Park JH. Combination chemotherapy with methotrexate and vinblastine for surgically unresectable, aggressive fibromatosis. Jpn J Clin Oncol. 2016:46:845-9.

22. Penel N, Le Cesne A, Bui BN, et al. Chemotherapy in patients with desmoid tumors: a study from the French Sarcoma Group (FSG). Ann Oncol. 2012;23:182-6.

23. Gounder MM, Lefkowitz RA, Keohan ML, et al. Activity of sorafenib against desmoid tumor/deep fibromatosis. Clin Cancer Res. 2011;17:4082-90.

24. Szucs Z, Messiou C, Wong HH, et al. Pazopanib, a promising option for the treatment of aggressive fibromatosis. Anticancer Drugs. 2017. https://doi. org/10.1097/cad.0000000000000474.

25. Hughes DP, Kummar S, Lazar AJ. New, tolerable $\gamma$-secretase inhibitor takes desmoid down a notch. Clin Cancer Res. 2015;21:7-9.

26. Deyrup AT, Tretiakova M, Montag AG. Estrogen receptor-beta expression in extraabdominal fibromatoses: an analysis of 40 cases. Cancer. 2006;106:208-13.

27. Fiore M, Coppola S, Cannell AJ, et al. Desmoid-type fibromatosis and pregnancy: a multi-institutional analysis of recurrence and obstetric risk. Ann Surg. 2014;259:973-8.

28. Lotfi AM, Dozois RR, Gordon H, Hruska LS, Weiland LH, Carryer PW, Hurt RD. Mesenteric fibromatosis complicating familial adenomatous polyposis: predisposing factors and results of treatment. Int J Colorectal Dis. 1989:4.30-6.

29. Hansmann A, Vogel CAT, Unger A, Moeslein G. High dose tamoxifen and sulindac as first line treatment for desmoids tumors. Cancer. 2004; 100:612-20

30. Skapek SX, Anderson JR, Da Hill, et al. Safety and efficacy of high dose tamoxifen and sulindac for desmoids tumor in children: results of a Children's Oncology Group (COG) Phase II study. Paediatr Blood Cancer. 2013:60:1108-12.

31. Fiore M, Colombo C, Radaelli S, Callegaro D, et al. Hormonal manipulation with toremifene in sporadic desmoids-type fibromatosis. Eur J Cancer. 2015;51:2800-7.

32. Carthy JM, Sundqvist A, Heldin A, van Dam H, Kletsas D, Heldin CH, Moustakas A. Tamoxifen inhibits TGF- $\beta$-mediated activation of myofibroblasts by blocking non-smad signaling through ERK1/2. J Cell Physiol. 2015;230:3084-92.

33. Eisenhauer EA, Therasse $P$, Bogaerts J, et al. New response evaluation criteria in solid tumors: re-vised RECIST guideline (version 1.1). Eur J Cancer. 2009:45:228-47.

34. Murphey MD, Ruble CM, Tyszko SM, Zbojniewicz AM, Potter BK, Miettinem M. From the archives of the AFIP: musculoskeletal fibromatosesradiologic-pathologic correlation. RadioGraphics. 2009;29:2143-73.

35. Walker EA, Petscavage JM, Brian PL, et al. Imaging features of superficial and deep fibromatoses in the adult population. Sarcoma. 2012;2012:215810.

36. Lee JC, Thomas JM, Phillips S, Fisher C, Moskovic E. Aggressive fibromatosis: MRI features with pathologic correlation. AJR. 2006:186:247-54.

37. Dinauer PA, Brixey CJ, Moncur JT, Fanburg-Smith JC, Murphey MD. Pathologic and MR imaging features of benign fibrous soft-tissue tumours in adults. Radiographics. 2007;27:173-87

38. Sundaram M, McGuire MH, Schajowicz F. Soft tissue masses: histologic basis for decreased signal (short T2) on T2-weighted MR images. AJR. 1987;148:1247-50.

39. Otero S, Moskovic EC, Strauss DC, Benson C, Miah AB, Thway K. Desmoidtype fibromatosis. Clin. Radiology. 2015;70:1038-45. 
40. Castellazzi G, Vanel D, Le Cesne A, et al. Can the MRI signal of aggressive fibromatosis be used to predict its behavior? Eur J Radiol. 2009;69:222-9.

41. Kamali F, Wang W-L, Guadagnolo BA, et al. MRI may be used as a prognostic indicator in patients with extra-abdominal desmoid tumours. $\mathrm{Br}$ J Radiol. 2016;89:20150308.

42. Rodriguez-Wallberg KA, Oktay K. Fertility preservation in women with breast cancer. Clin Obstet Gynecol. 2010;53:753-62.

43. Weinblatt ME, Keystone EC, Furst DE, et al. Adalimumab, a fully human anti-tumor necrosis factor a monoclonal antibody, for the treatment of rheumatoid arthritis in patients taking concomitant methotrexate. Arthritis Rheum. 2003:48:35-42.

44. Fiore M, Rimareix F, Mariani L, et al. Desmoid type fibromatosis: a front line conservative approach to select patients for surgical treatment. Ann Surg Oncol. 2009;16:2587-93.

45. Brooks MD, Ebbs SR, Colletta AA, Baum M. Desmoid tumors treated with triphenylethylenes. Eur J Cancer. 1992;28A:1014-8.

46. Quast DR, Schneider R, Burdzik E, Hoppe S, Molsein G. Long-term outcome of sporadic and FAP-associated desmoid tumors treated with high-dose selective estrogen receptor modulators and sulindac: a single-center long-term observational study in 134 patients. Fam Cancer. 2016;15:31-40

47. Hial V, Horakova Z, Shaff FE, Beaven MA. Alteration of tumor growth by aspirin and indomethacin: studies with two transplantable tumors in mouse. Eur J Pharmacol. 1976:37:367-76.

48. Sheth PJ, Del Moral S, Wilky BA, et al. Desmoid fibromatosis: MRI features of response to systemic therapy. Skeletal Radiol. 2016:45:1365-73.

49. Kasper B, Dimitrakopoulou-Strauss A, Pilz LR, Strauss LG, Sachpekidis C, Hohenberger P. Positron emission tomography as a surrogate marker for evaluation of treatment response in patients with desmoid tumors under therapy with imatinib. Biomed Res Int. 2013;2013:389672

50. Bonvalot $S$, Ternès $N$, Fiore $M$, et al. Spontaneous regression of primary abdominal wall desmoid tumors: more common than previously thought. Ann Surg Oncol. 2013;20:4096-102.
Ready to submit your research? Choose BMC and benefit from:

- fast, convenient online submission

- thorough peer review by experienced researchers in your field

- rapid publication on acceptance

- support for research data, including large and complex data types

- gold Open Access which fosters wider collaboration and increased citations

- maximum visibility for your research: over $100 \mathrm{M}$ website views per year

At BMC, research is always in progress.

Learn more biomedcentral.com/submissions 